\title{
SINGULAR FOLIATIONS WITH EHRESMANN CONNECTIONS AND THEIR HOLONOMY GROUPOIDS
}

\author{
NINA I. ZHUKOVA \\ Department of Mechanics and Mathematics \\ Nizhny Novgorod State University \\ 23 Gagarin Avenue, 603950 Nizhny Novgorod, Russia \\ E-mail: n.i.zhukova@rambler.ru
}

\begin{abstract}
We introduce topological $\mathcal{Q}$-holonomy groupoids for singular foliations $(M, \mathcal{F})$ with an Ehresmann connection $\mathcal{Q}$ using $\mathcal{Q}$-holonomy groups, which have a global character. We show advantage of our groupoids over known ones.
\end{abstract}

1. Introduction. The concept of Ehresmann connection for foliations was introduced by Blumenthal and Hebda [3] as a natural generalization of Ehresmann connections for submersions.

The construction of Ehresmann connection is based on the existence of transfers of horizontal curves along vertical curves through the vertical horizontal homotopy. Note that this homotopy has already been used in global differential geometry (see, e. g., [8], [10], [19]).

We extend the concept of Ehresmann connection to foliations $(M, \mathcal{F})$ with singularities in the sense of Stefan [20] and Sussmann [21] (Section 3).

Transversally complete singular Riemannian and totally geodesic foliations with complete induced Riemannian metrics on the leaves have natural Ehresmann connections.

We define an Ehresmann connection $\mathcal{Q}$ of singular foliations $(M, \mathcal{F})$ as a generalized distribution $\mathcal{Q}$ on $M$, which is transverse to $\mathcal{F}$, and a vertical horizontal property is satisfied [34]. This property allows to transfer an integral curve $\sigma$ of $\mathcal{Q}$ (called horizontal) along admissible curves (called vertical) lying in the leaf $L=L(\sigma(0))$ of $\mathcal{F}$. Unlike the regular case this transfer is not unique in general (Remark 1).

2000 Mathematics Subject Classification: Primary 53C12; Secondary 57R30.

Key words and phrases: foliation, foliation with singularities, Ehresmann connection, holonomy groupoid, Riemannian singular foliation.

Research supported by Russian Foundation for Basic Research, grant N 06-01-00331-a.

The paper is in final form and no version of it will be published elsewhere. 
Therefore we define the $* \mathcal{Q}$-holonomy group $* H_{\mathcal{Q}}(L, a)$ of an arbitrary leaf $L$ of $(M, \mathcal{F})$ as the transformation group of a quotient set $\Omega_{a} / \rho$ of the set $\Omega_{a}$ of horizontal curves with the origin in $a$. We stress that this group has a global character (Section 4).

For a singular Riemannian foliation this transfer keeps lengths of horizontal curves. We use this property and prove a criterion of local stability of leaves of singular foliations (Section 11, Theorem 5).

The concept of a topological holonomy groupoid was introduced by C. Ehresmann [6]. A new approach to the concept of the holonomy groupoid was found by J. Pradines [16], [17]. He used germs of local transverse isomorphisms of foliation and defined the notion of a differentiable holonomy groupoid. H. E. Winkelnkemper [23] suggested an equivalent construction and called it the graph $G(\mathcal{F})$ of the foliation $\mathcal{F}$.

Under some assumptions holonomy groups of singular foliations were defined by M. Bauer [1] and P. Dazord [4]. For Stefan foliation with only tractable singular leaves a topological holonomy groupoid was defined by H. Suzuki [22]. B. Bigonnet and J. Pradines [2] indicated another construction of a graph of a singular foliation.

We introduce a graph $G(\mathcal{F}, Q)$ for regular foliation $(M, \mathcal{F})$ with an Ehresmann connection $\mathcal{Q}$ [28] (see also [30], [31]) and prove that this graph has important advantages over the usual graph $G(\mathcal{F})$. In particular, our graph is always a Hausdorff manifold, and its projections onto $M$ form a locally trivial fibration. It allows to apply this graph in investigation of a topology of regular foliations admitting an Ehresmann connection.

R. A. Wolak [24] proved that a regular foliation $\mathcal{F}$ with an Ehresmann connection has no vanishing cycles. Relations between sets with Hausdorff and non-Hausdorff graphs of suspended foliations were investigated by G. Chubarov and the author [32].

The main goal of this paper is a construction of a topological groupoid of a singular foliation with an Ehresmann connection. The global character of the $* \mathcal{Q}$-holonomy group $* H_{\mathcal{Q}}(L, a)$ allows us to introduce a topological holonomy groupoid $* G_{\mathcal{Q}}(\mathcal{F})$ using our $* \mathcal{Q}$-holonomy groups (Section 6, Proposition 3).

We define a generalized Ehresmann connection of a singular foliation and $\mathcal{Q}$-holonomy group $H_{\mathcal{Q}}(L, a)$ of a leaf $L$, and by contrast to the group $* H_{\mathcal{Q}}(L, a)$ the set of horizontal curves is formed by curves for which only endpoints may be singular (Section 8).

Using $\mathcal{Q}$-holonomy groups $H_{\mathcal{Q}}(L, a)$ we define a holonomy groupoid $G_{\mathcal{Q}}(\mathcal{F})$ of a singular foliation $(M, \mathcal{F}, Q)$ with generalized Ehresmann connection $\mathcal{Q}$. Under natural assumptions we prove some properties of the groupoid $G_{\mathcal{Q}}(\mathcal{F})$, in particular the property of the topological space of $G_{\mathcal{Q}}(\mathcal{F})$ to be Hausdorff (Section 9, Theorem 2).

As corollary, $* G_{\mathcal{Q}}(\mathcal{F})=G_{\mathcal{Q}}(\mathcal{F})=G(\mathcal{F}, Q)$ is Hausdorff for regular foliation with an Ehresmann connection $(M, \mathcal{F}, Q)$, where $G(\mathcal{F}, Q)$ is the graph mentioned above.

In particular we prove that the holonomy groupoid $G_{\mathcal{Q}}(\mathcal{F})$ of an orbit-like transversally complete singular Riemannian foliation $(M, \mathcal{F}, Q)$ with a natural Ehresmann connection $\mathcal{Q}=(T \mathcal{F})^{\perp}$ is Hausdorff (Section 12, Corollary 4).

We hope that the groupoid $G_{\mathcal{Q}}(\mathcal{F})$ will be applied to singular foliations with transversal geometric structures. Nowadays the most studied class of these foliations is the class of Riemannian foliations ([12]). R.A. Wolak [25] introduces and investigates singular foliations on stratified manifolds admitting adapted linear connections. 
In recent work of C. Debord [5] a Lie groupoid of a singular foliation was constructed by a new method using local Lie groupoids and their equivalences without holonomy groups. As indicated in [5] the foliation $\mathcal{F}$ of $\mathbf{R}^{3}$ by concentric spheres with unique singular leaf 0 has no holonomy groupoid in the sense of C. Debord. This foliation $\left(\mathbf{R}^{3}, \mathcal{F}\right)$ is a Riemannian foliation with the natural Ehresmann connection $\mathcal{Q}=(T \mathcal{F})^{\perp}$. Thus the holonomy groupoids $* G_{\mathcal{Q}}(\mathcal{F})$ and $G_{\mathcal{Q}}(\mathcal{F})$ are well defined, and according to Corollary 4 the topological space $G_{\mathcal{Q}}(\mathcal{F})$ is Hausdorff.

A. Piątkowski [14] constructed the holonomy group of a leaf of a singular foliation which can be considered as an analogous of a germ holonomy group of a regular foliation. He proved a local transverse stability of a compact leaf with the trivial holonomy group [14].

Under an additional assumption the local stability in the sense of Ehresmann of a compact leaf with a finite holonomy group was proved by P. Dazord [4].

We state a theorem about global stability of a compact leaf $L$ of maximal dimension with a finite holonomy group $H_{\mathcal{Q}}(L)$ (or a finite fundamental group $\pi_{1}(L)$ ), which belongs to the author [34] (Section 10).

2. Foliations with singularities. We assume that all manifolds under consideration are of class $C^{r}, r \geq 1$, connected, second countable, and Hausdorff, unless otherwise stated. All neighbourhoods are open. Paths, curves and maps are piecewise differentiable.

Let $M$ be an $n$-dimensional manifold. A map $\mathcal{T}$ assigning to each point $x \in M$ a $p(x)$ dimensional subspace $\mathcal{T}_{x}$ in the tangent space $T_{x} M$, is called a generalized distribution on $M$. The distribution $\mathcal{T}$ is said to be differentiable if for any vector $Y \in \mathcal{T}_{x}$ there exists a differentiable vector field $X$ in a neighbourhood $U_{x}$ of $x$ such that $X_{x}=Y, X_{y} \in \mathcal{T}_{y}$ for any $y \in U_{x}$.

An immersed submanifold $L$ of $M$ is called an integral manifold of a generalized distribution $\mathcal{T}$ if $T_{x} L=\mathcal{T}_{x}$ for any $x \in L$. An integral manifold $L$ is said to be maximal if $L$ is connected and coincides with each connected integral manifold containing $L$. If through each point $x \in M$ an integral manifold passes, then the generalized distribution $\mathcal{T}$ is said to be integrable.

The family $\mathcal{F}=\left\{L_{\alpha} \mid \alpha \in J\right\}$ of maximal integral manifolds $L_{\alpha}$ of a generalized integrable distribution $\mathcal{T}$ is called a differentiable foliation with singularities. $L_{\alpha}$ considered as a submanifold in $M$ is called a leaf of the foliation $\mathcal{F}$.

H. Sussmann proved that a $C^{\infty}$-smooth generalized distribution $\mathcal{T}$ is integrable if and only if $\mathcal{T}$ is involutive (a generalization of Frobenius' theorem) [21].

Let $(M, \mathcal{F})$ be a differentiable foliation with singularities. It is known [4] that at each point $x \in M$ there exists a chart $(W, \varphi)$ such that

$\left(F_{1}\right) \varphi(W)=U \times V$, where $U$ is a neighbourhood of the origin in $\mathbf{R}^{p}, V$ is a neighbourhood of the origin in $\mathbf{R}^{q}, p=p(x)$ is the dimension of the central leaf $L(x)$ passing through $x$, and $q=n-p$;

$\left(F_{2}\right) \varphi(x)=(0,0) \in U \times V$;

$\left(F_{3}\right)$ for any leaf $L \in \mathcal{F}, \varphi(L \cap W)=U \times l$, where $l:=\left\{v \in V \mid \varphi^{-1}(0, v) \in L\right\}$. 
A chart $(W, \varphi)$ satisfying $\left(F_{1}\right)-\left(F_{3}\right)$ is said to be fibred at $x$, and $W$ is called a fibred neighbourhood. Also we call $x$ the center of this chart.

J. Kubarski [11] shown that Stefan's definition of a singular foliation is equivalent to the existence of a partition $\mathcal{F}=\left\{L_{\alpha} \mid \alpha \in J\right\}$ of $M$ into connected immersed submanifolds $L_{\alpha}$ such that for each $x \in M$, there is a fibred chart at $x$ with respect to $\mathcal{F}$. From this fact and Dazord's result [4] follows the equivalence of Stefan's and Sussmann's approaches to the notion of a $C^{\infty}$-smooth singular foliation.

Let $(W, \varphi)$ be a fibred chart, $\varphi(W)=U \times V$. Without loss of generality, we can assume that $U$ and $V$ are neighbourhoods homeomorphic to $\mathbf{R}^{p}$ and $\mathbf{R}^{q}$, respectively. In this work we assume that charts under consideration are fibred.

The arc-wise connected component of $W \cap L_{\alpha}$ will be called a local leaf of the leaf $L_{\alpha}$ in chart or in the neighbourhood $W$ and will be denoted by $l_{y}^{W}$, where $y \in W \cap L_{\alpha}$.

Note that, in the fibred neighbourhood $W_{x}$ of $x$, the dimension $p(y)$ of any leaf $L(y)$, $y \in W_{x}$ is not less than $p(x)$. Hence the leaf dimension function $p(x), x \in M$ is lower semi-continuous.

Since $p(x) \leq n, x \in M$, where $n=\operatorname{dim} M$, there exists the maximal dimension $p_{0}$ of leaves of foliation $\mathcal{F}$ with singularities. A point $x$ is said to be regular if a leaf of dimension $p_{0}$ passes through $x$. If a point $x \in M$ is not regular, $x$ is said to be singular. If $\operatorname{dim} L=p_{0}$, the leaf $L$ is said to be regular, otherwise $L$ is called a singular leaf. A foliation whose leaves have constant dimension is called regular. Therefore regular foliations form a subclass of the class of foliations with singularities.

Let us denote by $M^{0}$ the union of leaves of maximal dimension $p_{0}$. If $x \in M^{0}$, and $(W, \varphi)$ is a fibred chart at $x$, then for any $y \in W$ the leaf $L(y)$ also has dimension $p_{0}$, hence $W \subset M^{0}$. Thus the union $M^{0}$ of regular leaves is an open subset in $M$.

3. Ehresmann connection for foliations with singularities. A generalized distribution $\mathcal{Q}$ on the manifold $M$ is said to be transversal to the foliation $\mathcal{F}$ if on $M$ there exists a Riemannian metric $g$ such that $\mathcal{Q}_{x}$ is the orthogonal complement to the space $\mathcal{T}_{x}$ tangent to the leaf $L(x)$ in the Euclidean vector space $\left(T_{x} M, g_{x}\right)$, i.e., for any $x \in M$

$$
T_{x} M=\mathcal{T}_{x} \oplus \mathcal{Q}_{x},
$$

where $\oplus$ is the orthogonal sum. The subspace $\mathcal{Q}_{x}, x \in M$, and vectors in $\mathcal{Q}$ will be called horizontal. A piecewise differentiable curve $\sigma$ is said to be horizontal if its tangent vectors are horizontal. The distribution $\mathcal{T}$ tangent to the leaves of $\mathcal{F}$ is said to be vertical. A curve $h$ is said to be vertical if $h$ lies in a leaf of $\mathcal{F}$.

A vertical horizontal homotopy (v.h.h.) is a piecewise differentiable map $H: I_{1} \times I_{2} \rightarrow$ $M$, where $I_{1}=I_{2}=[0,1]$ is such that for any $(s, t) \in I_{1} \times I_{2}$ the curve $\left.H\right|_{I_{1} \times\{t\}}$ is horizontal and the curve $\left.H\right|_{\{s\} \times I_{2}}$ is vertical (see Fig. 1). The pair of curves $\left(\left.H\right|_{I_{1} \times\{0\}},\left.H\right|_{\{0\} \times I_{2}}\right)$ is called the base of the v.h.h. A pair of paths $(\sigma, h)$ such that $\sigma(0)=h(0), \sigma$ is a horizontal path, and $h$ is a vertical path, is said to be admissible.

Definition 1. A generalized distribution $\mathcal{Q}$ transversal to the foliation $\mathcal{F}$ with singularities is called an Ehresmann connection for $\mathcal{F}$ if for any admissible pair of paths $(\sigma, h)$ 


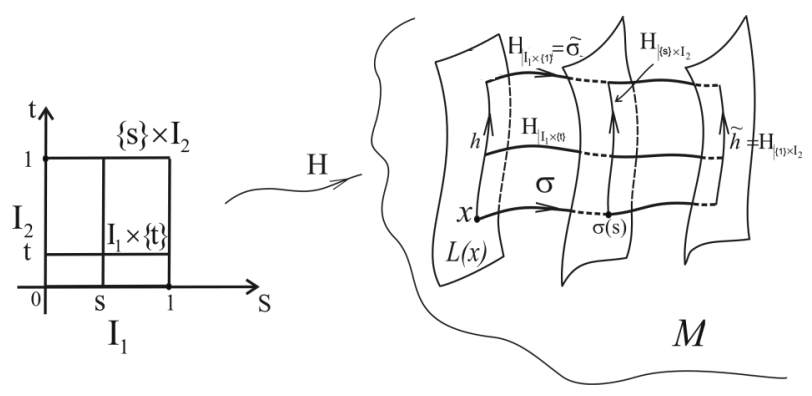

Fig. 1. A vertical horizontal homotopy with the base $(\sigma, h)$

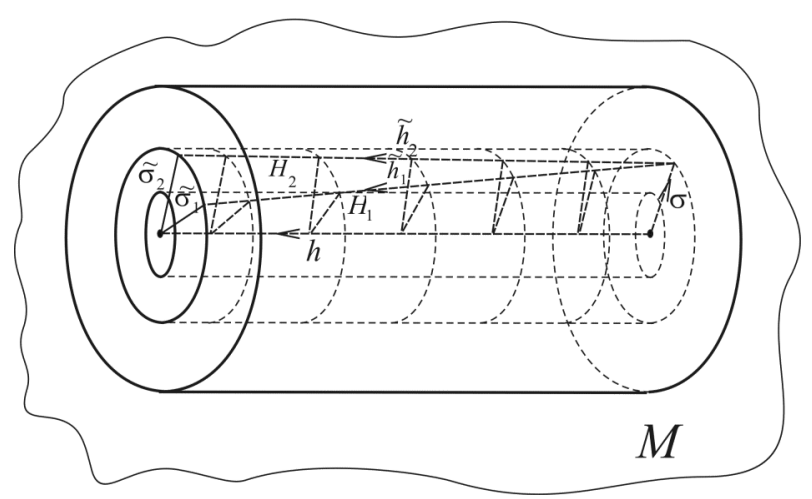

Fig. 2. Different vertical horizontal homotopies $H_{1}$ and $H_{2}$ of the singular Riemannian foliation with the same base $(\sigma, h)$

there exists a v.h.h. with the base $(\sigma, h)$. A foliation $(M, \mathcal{F})$ with singularities admitting an Ehresmann connection $\mathcal{Q}$ is denoted by $(M, \mathcal{F}, \mathcal{Q})$.

Let $H$ be a v.h.h. with the base $(\sigma, h)$, and $\tilde{\sigma}:=\left.H\right|_{I_{1} \times\{1\}}, \tilde{h}:=\left.H\right|_{\{1\} \times I_{2}}$. We say that the curve $\tilde{\sigma}$ is obtained from $\sigma$ by transfer along $h$ via the v.h.h. $H$ and denote it by $\sigma \underset{h}{\stackrel{H}{\longrightarrow}}>\tilde{\sigma}$ or by $\sigma \stackrel{H}{\longrightarrow}>\tilde{\sigma}$ and similarly $h \underset{\sigma}{\stackrel{H}{\longrightarrow}}>\tilde{h}$ or $h \stackrel{H}{\longrightarrow}>\tilde{h}$.

Note that, in general, an Ehresmann connection $\mathcal{Q}$ is not a differentiable generalized distribution. However, $\mathcal{Q}$ possesses a "generalized differentiability" property because $\mathcal{Q}$ is the orthogonal complement to the differentiable generalized distribution $\mathcal{T}$ tangent to $\mathcal{F}$ (with respect to a Riemannian metric $g$ on $M$ ).

Also note that our definition of Ehresmann connection for foliations with singularities naturally generalizes the definition of Ehresmann connection for regular foliations [3].

REMARK 1. If $(M, \mathcal{F}, \mathcal{Q})$ is a regular foliation with an Ehresmann connection, it is well known that there exists a unique vertical horizontal homotopy $H$ with the given base $(\sigma, h)$. This is not true in general for singular foliations $(M, \mathcal{F}, \mathcal{Q})$. Hence transfer of $\sigma$ along $h$ is a multivalued function (see Fig. 2).

4. $* \mathcal{Q}$-holonomy group for foliation with singularities. For any foliation with singularities admitting an Ehresmann connection $\mathcal{Q}$ we introduce an $* \mathcal{Q}$-holonomy group. 
Let $\Omega_{a}$ be the set of horizontal curves with initial point $a$. Take an equivalence relation $\rho$ on $\Omega_{a}$ as follows. Curves $\sigma$ and $\sigma^{\prime}$ in $\Omega_{a}$ are said to be $\rho$-equivalent if there exist a vertical loop $h_{0}$ at $a$ homotopic to the constant loop $e_{a}$ in $L(a)$ and a v.h.h. $H$ with the base $\left(\sigma, h_{0}\right)$ such that $\left.H\right|_{I_{1} \times\{1\}}=\sigma^{\prime}$ (Fig. 3).

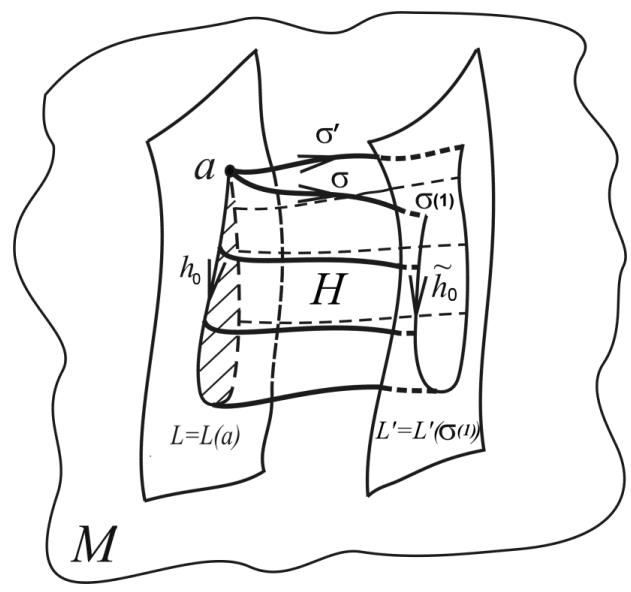

Fig. 3. The curves $\sigma$ and $\sigma^{\prime}$ are $\rho$-equivalent

One can directly check that $\rho$ is an equivalence relation in $\Omega_{a}$. Let us denote by $[\sigma]_{\rho}$ the $\rho$-equivalence class of a curve $\sigma$, and by $\Omega_{a} / \rho$ the set of equivalence classes.

Proposition 1. The map

$$
\Phi_{a}: \Omega_{a} / \rho \times \pi_{1}(L, a) \rightarrow \Omega_{a} / \rho:\left([\sigma]_{\rho},[h]\right) \mapsto[\tilde{\sigma}]_{\rho},
$$

where $[h] \in \pi_{1}(L, a), H$ is a v.h.h. with the base $(\sigma, h)$, and $\sigma \stackrel{H}{\longrightarrow}>\tilde{\sigma}$, defines a right action of the fundamental group $\pi_{1}(L, a)$ of $L(a)$ on the quotient set $\Omega_{a} / \rho$.

Proof. Let us demonstrate that $\Phi_{a}$ is correctly defined, i.e., does not depend on

1) the choice of the loop $h$ in the class $[h] \in \pi_{1}(L, a)$ and the choice of the v.h.h. $H$ with the base $(\sigma, h)$;

2) the choice of $\sigma$ in the class $[\sigma]_{\rho}$.

1. In a leaf $L$ let us take paths $h$ and $h^{\prime}$ joining $a$ with $b$. Suppose that $h$ is homotopic to $h^{\prime}$ in $L$, and $\sigma$ is a curve in $\Omega_{a}$. Let $H, H^{\prime}$ be v. h. hs with the bases $(\sigma, h)$ and $\left(\sigma, h^{\prime}\right)$, respectively, and $\sigma \stackrel{H}{\longrightarrow}>\tilde{\sigma}, \sigma \stackrel{H^{\prime}}{\longrightarrow}>\sigma^{\prime}$. Let us prove that $[\tilde{\sigma}]_{\rho}=\left[\sigma^{\prime}\right]_{\rho}$. Since $h$ is homotopic to $h^{\prime}$ in $L$, the loop $\phi_{0}:=h^{-1} h^{\prime}$ is homotopic to the constant path $e_{b}$ in $L$. Then we set

$$
K(s, t):= \begin{cases}H(s, 1-2 t), & \text { if }(s, t) \in I_{1} \times\left[0, \frac{1}{2}\right] ; \\ H^{\prime}(s, 2 t-1), & \text { if }(s, t) \in I_{1} \times\left[\frac{1}{2}, 1\right],\end{cases}
$$

and obtain that $K$ is a v.h.h. with the base $\left(\tilde{\sigma}, \phi_{0}\right)$ such that $\left.K\right|_{I_{1} \times\{1\}}=\sigma^{\prime}$. Hence $[\tilde{\sigma}]_{\rho}=\left[\sigma^{\prime}\right]_{\rho}$.

2. Let $\sigma \in \Omega_{a}, h$ be a vertical loop at $a$, and $H$ be a v.h.h. with the base $(\sigma, h)$. Take any path $\sigma^{*} \in[\sigma]_{\rho}$. Then there exists a v.h.h. $K$ with the base $\left(\sigma^{*}, h_{0}\right)$, where 
$\left[h_{0}\right]=\left[e_{a}\right] \in \pi_{1}(L, a)$, which provides the $\rho$-equivalence between $\sigma^{*}$ and $\sigma$, i.e., $K(s, 0)=$ $\sigma^{*}(s), K(s, 1)=\sigma(s), s \in I_{1}$, and $K(0, t)=h_{0}(t), t \in I_{2}$. Let us define the map $H^{*}: I_{1} \times I_{2} \rightarrow M:$

$$
H^{*}(s, t):= \begin{cases}K(s, 2 t), & \text { if }(s, t) \in I_{1} \times\left[0, \frac{1}{2}\right] ; \\ H(s, 2 t-1), & \text { if }(s, t) \in I_{1} \times\left[\frac{1}{2}, 1\right] .\end{cases}
$$

Since $K(s, 1)=H(s, 0)=\sigma(s), s \in I$, we have that $H^{*}$ is a v.h.h. as well as $K$ and $H$. The pair of paths $\left(\sigma^{*}, h_{0} h\right)$ is the base of $H^{*}$. In addition, $\sigma^{*} \stackrel{H^{*}}{\longrightarrow}>\tilde{\sigma}$. Since $\left[h_{0} h\right]=$ $[h] \in \pi_{1}(L, a)$, from the definition of $\Phi_{a}$ we get that $\Phi_{a}\left(\left[\sigma^{*}\right]_{\rho},[h]\right)=[\tilde{\sigma}]_{\rho}$. Thus the map $\Phi_{a}$ is correctly defined.

One can easily check that $\Phi_{a}$ defines a right action of $\pi_{1}(L, a)$ on $\Omega_{a} / \rho$.

Definition 2. The kernel of the action $\Phi_{a}, * K_{\mathcal{Q}}(L, x):=\operatorname{ker} \Phi_{a}=\left\{[h] \in \pi_{1}(L, a) \mid\right.$ $\left.\left(\forall[\sigma]_{\rho} \in \Omega_{a} / \rho\right) \Phi_{a}\left([\sigma]_{\rho},[h]\right)=[\sigma]_{\rho}\right\}$, is a normal subgroup in $\pi_{1}(L, a)$. Hence we have the quotient group

$$
* H_{\mathcal{Q}}(L, a):=\pi_{1}(L, a) / \operatorname{ker} \Phi_{a} .
$$

Let us call the group $* H_{\mathcal{Q}}(L, a)$ the $* \mathcal{Q}$-holonomy group for the singular foliation with an Ehresmann connection $(M, \mathcal{F}, \mathcal{Q})$.

As in [15], the $*$ indicates that $* H_{\mathcal{Q}}(L, a)$ is a holonomy group of foliation with singularities, which differs from the known holonomy groups. For a regular foliation each equivalence class $[\sigma]_{\rho}$ consists of one horizontal curve $\sigma$, hence the set $\Omega_{a} / \rho$ is bijective to the set $\Omega_{a}$. In this case $* H_{\mathcal{Q}}(L, a)$ coincides with the group $H_{\mathcal{Q}}(L, a)$, which was defined in [3] (and was called the $\mathcal{Q}$-holonomy group in [28], [30]). It is not difficult to show that the following statement takes place.

Proposition 2. For any points a and $b$ in a leaf $L$ of the foliation $(M, \mathcal{F}, \mathcal{Q})$ there exists an isomorphism $* H_{\mathcal{Q}}(L, a) \rightarrow * H_{\mathcal{Q}}(L, b)$, which is unique up to inner automorphisms of these groups.

By Proposition 2 we can speak about the $* \mathcal{Q}$-holonomy group of a leaf $L$ with understanding that this group is the algebraic group $* H_{\mathcal{Q}}(L)$ which does not depend on $a \in L$. We say that a leaf $L$ has finite $* \mathcal{Q}$-holonomy group if this group is finite.

5. Subordinated charts. Let $(M, \mathcal{F}, Q)$ be a singular foliation with Ehresmann connection. Let us consider a fibred chart $(W, \varphi)$ at a point $a, \varphi(W)=U \times V$. Then $\mathcal{F}_{W}:=\left\{\varphi^{-1}(U \times\{y\}) \mid y \in V\right\}$ is a simple foliation in $W$, and let $\pi: W \rightarrow W / \mathcal{F}_{W}$ be the projection on the space of leaves. We say that the distribution $\mathcal{Q}$ has the property of local transversal projectability if at an arbitrary point $a \in M$ there exists a fibred chart $(W, \varphi)$ with center $a$ such that, for an arbitrary curve $\sigma \in \Omega_{a}$ in $W$ at each point $y$ in the local leaf $l_{a}^{W}$ there exists a curve $\sigma_{y} \in \Omega_{y}$ smoothly depending on $y$ and satisfying $\pi \circ \sigma_{y}=\pi \circ \sigma$.

After this we assume that for a foliation $(M, \mathcal{F}, Q)$ the Ehresmann connection $\mathcal{Q}$ has the property of local transversal projectability.

Definition 3. Let $(W, \varphi)$ and $(V, \psi)$ be two fibred charts with center in $a$ and $b \in L(a)$ respectively of singular foliation $(M, \mathcal{F})$. We say that $\operatorname{chart}(V, \psi)$ is subordinated to the 
chart $(W, \varphi)$ if the following conditions are satisfied:

(i) $\varphi(W)=U_{1} \times V_{1}, \psi(V)=U_{2} \times V_{2}$, with $0 \in V_{2} \subset V_{1} \subset \mathbf{R}^{q}, q=n-p, p=p(a)$.

(ii) Let $L$ be an arbitrary leaf of $\mathcal{F}$ such that $L \cap V \neq \emptyset$. Then for any connected component $c(L \cap V)$ of $L \cap V$ the set $\varphi^{-1}(\psi(c(L \cap V)))$ belongs to some connected component of the intersection $L \cap W$.

For regular foliations the notion of subordinated charts was introduced by R. Palais [13].

Let $a, b$ be points of the same leaf $L \in \mathcal{F}$. Connect $a$ with $b$ by a path $h$ in $L$ and cover the set $h([0,1])$ by a finite chain of charts $\left(W_{k}, \varphi_{k}\right)$ with center at $a_{k}=h\left(t_{k}\right), t_{k} \in[0,1]$, $k=0, \ldots, m$, where $\left(W_{0}, \varphi_{0}\right)=(W, \varphi), a_{0}=a, a_{m}=b$. Without loss of generality we can assume that the sets $W_{k} \cap W_{k+1} \cap h([0,1])$ are connected and an arbitrary local leaf of $W_{k+1}$ intersects some local leaf of $W_{k}$.

By analogy with [13] using a local transversal containing a point $h\left(\tau_{1}\right) \in W_{1} \cap W_{2}$, $\tau_{1} \in[0,1]$, we change coordinates in the neighbourhood $W_{1}$ such that $\left(W_{1}, \varphi_{1}\right)$ becomes a subordinated chart to $\left(W_{0}, \varphi_{0}\right)$. If necessary we decrease $W_{1}$ to $V_{1} \subset W_{1}$ such that $\left(V_{1}, \psi_{1}\right)$ becomes a subordinated chart to $\left(W_{0}, \varphi_{0}\right)$ with center $a_{1}$.

After this we construct a chart $\left(V_{2}, \psi_{2}\right)$ with center $a_{2}$ subordinated to $\left(V_{1}, \psi_{1}\right)$. Remark that the relation of subordination is transitive, so $\left(V_{2}, \psi_{2}\right)$ is subordinated to $(W, \varphi)$. By analogy in several steps we construct a chart $(V, \psi)$ with center $b$ subordinated to $(W, \varphi)$. Thus we have the following theorem.

THEOREM 1. Let $(M, \mathcal{F})$ be a singular foliation and $(W, \varphi)$ be a fibred chart with center $a \in M$. For an arbitrary point $b \in L(a)$ there exists a fibred chart $(V, \psi)$ with center $b$ subordinated to the chart $(W, \varphi)$.

The analogous theorem for a regular foliation was proved by R. Palais [13].

Moving along $h^{-1}$ from $b$ to $a$ we can construct fibred charts $(V, \chi)$ and $\left(V^{\prime}, \chi^{\prime}\right)$ with centers $a$ and $b$ such that each of them is subordinated to the other. In this case we shall call $(V, \chi)$ and $\left(V^{\prime}, \chi^{\prime}\right)$ mutually subordinated charts.

REMARK 2. Let $(U, \varphi)$ and $(V, \psi)$ be mutually subordinated charts. From the property of transversal projectibility and construction of a subordinated chart it follows that for any admissible pair $(\sigma, h)$ with $\sigma$ lying in $U$, where $\sigma(0)=h(0)$ is the center of $U$, there exists such v.h.h. $H$ that $\sigma \stackrel{H}{\longrightarrow}>\tilde{\sigma}$, and $\tilde{\sigma}$ is contained in $V$.

6. Groupoid of $* \mathcal{Q}$-holonomy. Let $(M, \mathcal{F}, Q)$ be an arbitrary singular foliation with an Ehresmann connection $\mathcal{Q}$. We denote by $[h]$ the homotopic class of vertical paths containing $h$. Let $x, y$ be both in a leaf $L$ of $\mathcal{F}$. Denote by $A(x, y)$ the set of all piecewise smooth path from $x$ to $y$ in $L$. We use notations introduced above. Two paths $h_{1}, h_{2} \in$ $A(x, y)$ are called equivalent if and only if $\left[h_{1} \cdot h_{2}^{-1}\right] \in * K_{\mathcal{Q}}(L, x)$, where $\left[h_{1} \cdot h_{2}^{-1}\right] \in$ $\pi_{1}(L, x)$.

In other words $h_{1} \sim h_{2}$ if and only if the homotopy class $\left[h_{1} \cdot h_{2}^{-1}\right]$ of the loop $h_{1} \cdot h_{2}^{-1}$ acts on the factor set $\widetilde{\Omega}_{x}:=\Omega_{x} / \rho$ in the trivial way. We denote by $\langle h\rangle$ the equivalence class containing $h$. 
Definition 4. We call the set

$$
* G_{\mathcal{Q}}(\mathcal{F}):=\{(x,\langle h\rangle, y) \mid x \in M \wedge y \in L(x) \wedge h \in A(x, y)\}
$$

a graph of singular foliation $(M, \mathcal{F})$ with an Ehresmann connection $\mathcal{Q}$.

The equality

$$
(x,\langle h\rangle, y) \circ(z,\langle g\rangle, x):=(z,\langle g \cdot h\rangle, y),
$$

where $g \cdot h$ is the product of paths $g$ and $h$, defines a partial multiplication $\circ$ in $* G_{\mathcal{Q}}(\mathcal{F})$ relative to which $* G_{\mathcal{Q}}(\mathcal{F})$ is a groupoid called the $* \mathcal{Q}$-holonomy groupoid of this foliation.

The maps $p_{1}: * G_{\mathcal{Q}}(\mathcal{F}) \rightarrow M:(x,\langle h\rangle, y) \mapsto x$ and $p_{2}: * G_{\mathcal{Q}}(\mathcal{F}) \rightarrow M:(x,\langle h\rangle, y) \mapsto y$ are called the source and range maps or canonical projections.

Now we introduce a topology in $* G_{\mathcal{Q}}(\mathcal{F})$. Let $c=(a,\langle h\rangle, b)$ be any point in $* G_{\mathcal{Q}}(\mathcal{F})$ and $(W, \varphi)$ and $\left(W^{\prime}, \varphi^{\prime}\right)$ be arbitrary fibred neighbourhoods with centers $a$ and $b$, respectively. Suppose that there is a horizontal curve $\sigma$ in $W, \sigma \in \Omega_{a}$, and a transfer $\sigma \underset{h}{\stackrel{H}{\longrightarrow}}>\tilde{\sigma}$, such that there exists a curve $\sigma^{\prime} \in[\tilde{\sigma}]_{\rho} \in \widetilde{\Omega}_{b}, \sigma^{\prime}(I) \subset W^{\prime}$. It is equivalent to the existence of a path $h^{\prime}$ homotopic to $h$ and v.h.h. $K$ such that $\sigma \underset{h^{\prime}}{\longrightarrow}>\sigma^{\prime}, \sigma^{\prime}(I) \subset W^{\prime}$. Consider two arbitrary points $x, y$ in local leaves $l_{\sigma(1)}^{W}$ and $l_{\sigma^{\prime}(1)}^{W^{\prime}}$ respectively. We take paths $t_{x}$ in $l_{\sigma(1)}^{W}$ from $x$ to $\sigma(1)$ and $t_{y}$ in $l_{\sigma^{\prime}(1)}^{W^{\prime}}$ from $y$ to $\sigma^{\prime}(1)$. Let $h^{\prime} \stackrel{K}{\longrightarrow}>\tilde{h}$ and $g:=t_{x} \cdot \tilde{h} \cdot t_{y}^{-1}$. We have an element $(x,\langle g\rangle, y)$ that belongs to $* G_{\mathcal{Q}}(\mathcal{F})$.

Denote by $\mathcal{V}\left(c, W, W^{\prime}\right)$ the set of all elements $(x,\langle g\rangle, y)$ of $* G_{\mathcal{Q}}(\mathcal{F})$ defined in the indicated way by $c$ and the charts $(W, \varphi)$ and $\left(W^{\prime}, \varphi^{\prime}\right)$.

The following lemma will be useful later.

LEMMA 1. If products of vertical paths $h \cdot k$ and $k^{\prime} \cdot g$ have common ends, then the following equalities are equivalent

$$
\langle h \cdot k\rangle=\left\langle k^{\prime} \cdot g\right\rangle \text { and }\left\langle k^{\prime-1} \cdot h\right\rangle=\left\langle g \cdot k^{-1}\right\rangle .
$$

Proposition 3. The set $\Sigma:=\left\{\mathcal{V}\left(c, W, W^{\prime}\right)\right\}$ forms a base of some topology $\tau$ in $* G_{\mathcal{Q}}(\mathcal{F})$ relatively to which $* G_{\mathcal{Q}}(\mathcal{F})$ is a topological groupoid. A set $\mathbf{L}:=p_{1}^{-1}(L), L \in \mathcal{F}$ is open in the space $* G_{\mathcal{Q}}(\mathcal{F})$ if and only if $\Omega_{a}=\left\{e_{a}\right\}$, where $e_{a}$ is the constant path at $a \in L$.

Proof. Let $\mathcal{V}_{1}:=\mathcal{V}\left(c_{1}, W_{1}, W_{1}^{\prime}\right)$ and $\mathcal{V}_{2}:=\mathcal{V}\left(c_{2}, W_{2}, W_{2}^{\prime}\right)$ be two arbitrary sets from $\Sigma$, with $\mathcal{V}_{1} \cap \mathcal{V}_{2} \neq \emptyset$, and $c_{1}:=\left(a_{1},\langle h\rangle, b_{1}\right), c_{2}:=\left(a_{2},\langle g\rangle, b_{2}\right)$. Consider an arbitrary point $z \in \mathcal{V}_{1} \cap \mathcal{V}_{2}$. As $z \in \mathcal{V}_{1}$, so $z=\left(x,\left\langle t_{x} \cdot \tilde{h} \cdot t_{y}^{-1}\right\rangle, y\right)$ by construction of $\mathcal{V}_{1}$. As $z \in \mathcal{V}_{2}$, so $z=\left(x,\left\langle\tau_{x} \cdot \tilde{g} \cdot \tau_{y}^{-1}\right\rangle, y\right)$, with $x \in W_{1} \cap W_{2}, y \in W_{1}^{\prime} \cap W_{2}^{\prime}$, there are $\sigma \in \Omega_{a_{1}}, \gamma \in \Omega_{a_{2}}$, $h^{\prime} \in[h], g^{\prime} \in[g]$ and some transfers $h^{\prime} \underset{\sigma}{\stackrel{H}{\longrightarrow}}>\tilde{h}, g^{\prime} \underset{\gamma}{\stackrel{H^{\prime}}{\longrightarrow}}>\tilde{g}$ (Fig. 4).

As six points $x, \tilde{h}(0), \tilde{h}(1), \tilde{g}(0), \tilde{g}(1)$ and $y$ lie in the same leaf $L^{\prime}$ of $\mathcal{F}$, there exist pairwise mutually subordinated fibred neighbourhoods of these points contained in $W_{1} \cap$ $W_{2}$ and $W_{1}^{\prime} \cap W_{2}^{\prime}$, respectively. Denote the indicated neighbourhoods at $x$ and $y$ by $\tilde{W}_{1} \ni x$ and $\tilde{W}_{2} \ni y$.

Let us show the inclusion $\tilde{\mathcal{V}}:=\mathcal{V}\left(z, \tilde{W}_{1}, \tilde{W}_{2}\right) \subset \mathcal{V}_{1} \cap \mathcal{V}_{2}$. At first we check the inclusion $\tilde{\mathcal{V}} \subset \mathcal{V}_{1}$. Consider any point $w=\left(u,\left\langle t_{u} \cdot \tilde{k} \cdot t_{v}^{-1}\right\rangle, v\right)$ in $\tilde{\mathcal{V}}$, where $k:=t_{x} \cdot \tilde{h} \cdot t_{y}^{-1}$ and there exists $\delta \in \Omega_{x}$ in $\tilde{W}_{1}$ such that $\tilde{k}$ is the result of some transfer $k^{\prime} \underset{\delta}{\stackrel{H_{1}}{\longrightarrow}}>\tilde{k}, k^{\prime} \in[k]$, 


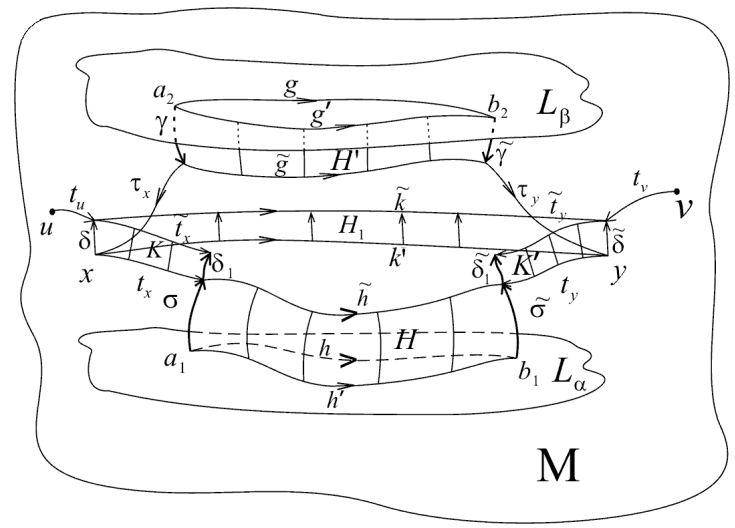

Fig. 4. A construction of $\tilde{\mathcal{V}} \subset \mathcal{V}_{1} \cap \mathcal{V}_{2}$

with $\delta \stackrel{H_{1}}{\longrightarrow}>\tilde{\delta}, \tilde{\delta} \in \Omega_{y}$ in $\tilde{W}_{2}$. According to the choice of $\tilde{W}_{1}, \tilde{W}_{2}$, there are some v.h.h. $\delta \underset{t_{x}}{\stackrel{K}{\longrightarrow}}>\delta_{1}, \tilde{\delta} \underset{t_{y}}{\stackrel{K^{\prime}}{\longrightarrow}}>\tilde{\delta}_{1}$ in $W_{1}$ and $W_{2}$ respectively. Denote $t_{x} \stackrel{K}{\longrightarrow}>\tilde{t}_{x}, t_{y} \stackrel{K^{\prime}}{\longrightarrow}>\tilde{t}_{y}$. We take Lemma 1 into account and have $\langle\tilde{h}\rangle=\left\langle t_{x}^{-1} \cdot k^{\prime} \cdot t_{y}\right\rangle$. Hence we may put $\tilde{h}^{\prime}:=t_{x}^{-1} \cdot k^{\prime} \cdot t_{y}$. Let us construct a v.h.h. $\tilde{K}$ by the formula

$$
\tilde{K}(s, t):=\left\{\begin{array}{l}
K(s, 1-4 t), t \in\left[0, \frac{1}{4}\right], \\
H_{1}(s, 4 t-1), t \in\left[\frac{1}{4}, \frac{1}{2}\right], \quad s \in[0,1], \\
K^{\prime}(s, 2 t-1), t \in\left[\frac{1}{2}, 1\right],
\end{array}\right.
$$

then $\delta_{1} \stackrel{\tilde{K}}{\longrightarrow}>\tilde{\delta}_{1}$ and $\tilde{h}^{\prime} \stackrel{\tilde{K}}{\longrightarrow}>\tilde{t}_{x}^{-1} \cdot \tilde{k} \cdot \tilde{t}_{y}=\hat{h}$ (Fig. 4).

So we have $w=\left(u,\left\langle\tau_{u} \cdot \hat{h} \cdot \tau_{v}^{-1}\right\rangle, v\right)$, where $\tau_{u}:=t_{u} \cdot \tilde{t}_{x}, \tau_{v}:=t_{v} \cdot \tilde{t}_{y}$. Therefore $w \in \mathcal{V}_{1}$.

The inclusion $\tilde{\mathcal{V}} \subset \mathcal{V}_{2}$ can be shown analogously. Thus $\Sigma$ is a base of some topology $\tau$ in $* G_{\mathcal{Q}}(\mathcal{F})$.

The continuity of the partial multiplication in $* G_{\mathcal{Q}}(\mathcal{F})$ can be checked in the usual way.

Now we assume the existence of a point $a \in M$ such that $\Omega_{a}=\left\{e_{a}\right\}$. In this case for any $b \in L=L(a)$ and arbitrary fibred charts $\left(W_{1}, \varphi_{1}\right)$ and $\left(W_{2}, \varphi_{2}\right)$ with centers $a, b$, respectively, the set $\mathcal{V}=\mathcal{V}\left(c, W_{1}, W_{2}\right), c=(a,\langle h\rangle, b)$, is formed by points $z=$ $\left(x,\left\langle t_{x} \cdot \tilde{h} \cdot t_{y}^{-1}\right\rangle, y\right)$, with $x \in l_{a}^{W_{1}}, y \in l_{b}^{W_{2}}$, i.e. $\mathcal{V} \subset \mathbf{L}:=p_{1}^{-1}(L)$. Therefore $\mathbf{L}$ is an open set in $* G_{\mathcal{Q}}(\mathcal{F})$.

Evidently, if $\mathbf{L}$ is an open set in $* G_{\mathcal{Q}}(\mathcal{F})$, then $\Omega_{a}=\left\{e_{a}\right\}, \forall a \in L$.

Let $G_{\mathcal{Q}}(\mathcal{F})$ be the graph of a regular foliation $(M, \mathcal{F}, Q)$ with an Ehresmann connection introduced in [28] ([30], [33]).

Proposition 4. Let $(M, \mathcal{F}, Q)$ be a regular foliation with an Ehresmann connection. Then the holonomy groupoid $* G_{\mathcal{Q}}(\mathcal{F})$ coincides with $G_{\mathcal{Q}}(\mathcal{F})$.

Proof. Let $\mathcal{V}=\mathcal{V}\left(c, W_{1}, W_{2}\right)$ be a neighbourhood of point $c=(a,\langle h\rangle, b)$ from the base $\Sigma$ of topology in $* G_{\mathcal{Q}}(\mathcal{F})$. Consider an arbitrary point $z=\left(x,\left\langle t_{x} \cdot \tilde{h} \cdot t_{y}^{-1}\right\rangle, y\right)$ in $\mathcal{V}$, defined by a path $h^{\prime} \in[h]$, a horizontal curve $\sigma$ in $W_{1}$ and v.h.h. $H$, with $\sigma \frac{H}{h^{\prime}}>\tilde{\sigma}$. Recall that 
$t_{x}$ and $t_{y}$ are paths in local leaves from $x$ to $\sigma(1)$ and $y$ to $\tilde{\sigma}(1)$ respectively. Let $\gamma$ be another horizontal curve in $W_{1}$ and $K$ be a v.h.h. with the base $\left(\gamma, h^{\prime \prime}\right), h^{\prime \prime} \in[h]$, with $\gamma \underset{h^{\prime \prime}}{\stackrel{K}{\longrightarrow}}>\tilde{\gamma}, h^{\prime \prime} \stackrel{K}{\longrightarrow}>\hat{h}$, and $z=\left(x,\left\langle\tau_{x} \cdot \hat{h} \cdot \tau_{y}^{-1}\right\rangle, y\right)$. In the regular case as all local leaves of fibred neighbourhoods are simply connected, the vertical paths $t_{x} \cdot \tilde{h} \cdot t_{y}^{-1}$ and $\tau_{x} \cdot \hat{h} \cdot \tau_{y}^{-1}$ are homotopy equivalent. Therefore the point $z$ does not depend on the choice of the curves $\gamma$ and $t_{x}, t_{y}$.

Thus in regular case the topology in $* G_{\mathcal{Q}}(\mathcal{F})$ defined by Proposition 3 coincides with the topology in the graph $G_{\mathcal{Q}}(\mathcal{F})$ introduced in the usual way [30].

\section{Simple transversal bifibrations}

Definition 5. Let $p_{i}: M \rightarrow B_{i}, i=1,2$, be a submersion with connected fibres. Suppose that the universal covering manifold of $M$ is a product of two manifolds $X_{1} \times X_{2}$. If the universal covering map $\kappa: X_{1} \times X_{2} \rightarrow M$ transforms fibres of the trivial fibrations $F_{i}$, where $F_{1}:=\left\{X_{1} \times\{v\} \mid v \in X_{2}\right\}, F_{2}:=\left\{\{u\} \times X_{2} \mid u \in X_{1}\right\}$, to corresponding fibres of the submersion $p_{i}$ then the quintuple $\left(M, p_{1}, p_{2}, B_{1}, B_{2}\right)$ is called a simple transversal bifibration by Ya. Shapiro and the author [18].

Remark that two submersions $p_{i}: M \rightarrow B_{i}, i=1,2$, of $M$ with connected fibres form a simple transversal bifibration if and only if the distribution $\mathcal{Q}_{1}$ tangent to fibres of $p_{2}: M \rightarrow B_{2}$ is an Ehresmann connection for the submersion $p_{1}: M \rightarrow B_{1}$.

It is well known that a diffeomorphism group $\Psi$ of manifold $N$ is a group of covering transformations of some regular covering map $f: N \rightarrow B$ onto the factor manifold $N / \Psi=$ $B$ if and only if $\Psi$ acts freely and properly discontinuously, i.e. for $x, y \in N$, where $y$ does not belong to the orbit $\Psi \cdot x$ of $x$, there exist two neighbourhoods $V$ and $W$ of $x$ and $y$ respectively such that for any $\psi \in \Psi, \psi \neq \mathrm{id}_{N}, \psi(V) \cap W=\emptyset$ holds. The next proposition follows from the work of Ya. Shapiro and the author [18].

Proposition 5. Let $\left(M, p_{1}, p_{2}, B_{1}, B_{2}\right)$ be a simple transversal bifibration. Let $x$ be an arbitrary fixed point in $M, x_{i}:=p_{i}(x) \in B_{i}$ and $Y_{i}:=p_{i}^{-1}\left(x_{i}\right)$, where $i=1,2$. Then we have the following:

(i) $p_{i}: M \rightarrow B_{i}$ is a locally trivial fibration with a standard fiber $Y_{i}$.

(ii) The map $p: M \rightarrow B_{1} \times B_{2}: x \mapsto\left(p_{1}(x), p_{2}(x)\right)$ is an regular covering map with the group $\Psi$ of covering transformations.

(iii) The restrictions $\left.p_{1}\right|_{Y_{2}}: Y_{2} \rightarrow B_{1}$ and $\left.p_{2}\right|_{Y_{1}}: Y_{1} \rightarrow B_{2}$ are regular covering maps with groups $\Psi_{2}$ and $\Psi_{1}$ of covering transformations respectively, and there exist group isomorphisms $\theta_{i}: \Psi \rightarrow \Psi_{i}$.

(iv) The action of $\Psi$ on the product $Y_{1} \times Y_{2}$ given by the rule

$$
\psi\left(y_{1}, y_{2}\right):=\left(\theta_{1}(\psi) y_{1}, \theta_{2}(\psi) y_{2}\right), \forall\left(y_{1}, y_{2}\right) \in Y_{1} \times Y_{2},
$$

is free and properly discontinuous. It defines a simple transversal bifibration $\left(\left(Y_{1} \times\right.\right.$ $\left.\left.Y_{2}\right) / \Psi, f_{1}, f_{2}, B_{1}, B_{2}\right)$, where $f_{i}: \Psi \cdot\left(y_{1}, y_{2}\right) \mapsto \Psi_{i} \cdot y_{i},\left(y_{1}, y_{2}\right) \in Y_{1} \times Y_{2}$, and there is a diffeomorphism $\Xi$ such that the following diagram is commutative 


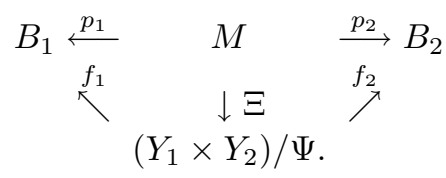

(v) The inclusion $j: Y_{i} \rightarrow M$ induces an isomorphism of the fundamental group $\pi_{1}\left(Y_{i}, x\right)$ onto a normal subgroup $G_{i i}$ of $\pi_{1}(M, x)$, and $\Psi$ is isomorphic to the factor group $\pi_{1}(M, x) /\left(G_{11} \times G_{22}\right)$.

Definition 6. The algebraic group $\Psi$ satisfying conditions of Proposition 5 is called the structure group of this bifibration.

The above notion is well defined, i.e. it does not depend on the choice of the canonical bifibration $\left(\left(Y_{1} \times Y_{2}\right) / \Psi, f_{1}, f_{2}, B_{1}, B_{2}\right)$ satisfying the conditions of Proposition 5 . Remark that each submersion $p_{i}$ forms the fibration with the structure group $\Psi$.

Definition 7. A bifibration $\left(M, p_{1}, p_{2}, B_{1}, B_{2}\right)$ is called symmetric if the fibrations $p_{i}: M$ $\rightarrow B_{i}, i=1,2$, are isomorphic (in the category of fibrations).

Proposition 6. Let $* G_{\mathcal{Q}}(\mathcal{F})$ be the holonomy groupoid of a singular foliation with an Ehresmann connection $(M, \mathcal{F}, Q)$ and $p_{1}, p_{2}: * G_{\mathcal{Q}}(\mathcal{F}) \rightarrow M$ be the canonical projections. Let $x$ be an arbitrary point in $M, L=L(x)$ and $\mathbf{L}:=p_{1}^{-1}(L)$. The quintuple $\left(\mathbf{L},\left.p_{1}\right|_{\mathbf{L}},\left.p_{2}\right|_{\mathbf{L}}, L, L\right)$ is a symmetric simple transversal bifibration with the structure group $\Psi$, that is isomorphic to the holonomy group $* H_{\mathcal{Q}}(L)$.

Proof. Let us introduce a smooth structure in $\mathbf{L}:=p_{1}^{-1}(L), L \in \mathcal{F}$. Let $c=(a,\langle h\rangle, b)$ be an arbitrary point in $\mathbf{L}$. Let $\left(W_{1}, \varphi_{1}\right)$ and $\left(W_{2}, \varphi_{2}\right)$ be an arbitrary mutually subordinated charts with centers $a, b$ respectively and $\mathcal{V}:=\mathcal{V}\left(c, W_{1}, W_{2}\right)$. Denote $\mathcal{U}:=\mathcal{V} \cap \mathbf{L}, p=\operatorname{dim} L$. The map $\chi: \mathcal{U} \rightarrow \mathbf{R}^{p} \times \mathbf{R}^{p}:\left(x,\left\langle t_{x} \cdot \tilde{h} \cdot t_{y}^{-1}\right\rangle, y\right) \mapsto\left(\varphi_{1}(x), \varphi_{2}(y)\right)$ is a homeomorphism onto $\varphi_{1}\left(W_{1}\right) \times \varphi_{2}\left(W_{2}\right)=\mathbf{R}^{p} \times \mathbf{R}^{p}$. The set of all these pairs $\{(\mathcal{U}, \chi)\}$ forms a $C^{\infty}$-atlas of $2 p$-dimensional manifold in $\mathbf{L}$. The restrictions $\left.p_{1}\right|_{\mathbf{L}}: \mathbf{L} \rightarrow L$ and $\left.p_{2}\right|_{\mathbf{L}}: \mathbf{L} \rightarrow L$ become submersions onto $L$.

We show that fibres of $p_{i}, i=1,2$, are arc-wise connected. Take two arbitrary points $c_{1}=(a,\langle h\rangle, b)$ and $c_{2}=(a,\langle g\rangle, d)$ in the fibre $p_{1}^{-1}(a)$. As the formula

$$
k_{t}(s):=\left\{\begin{array}{l}
h(s(1-2 t)), t \in\left[0, \frac{1}{2}\right], \\
g(s(2 t-1)), t \in\left[\frac{1}{2}, 1\right],
\end{array} \quad s \in[0,1]\right.
$$

defines a homotopy of maps $h$ and $g$ in $L$, so $\mu(t):=\left(a,\left\langle k_{t}\right\rangle,\left(h^{-1} \cdot g\right)(t)\right)$ is a path in $p_{1}^{-1}(a)$ from $c_{1}$ to $c_{2}$. Analogously, fibres of $p_{2}$ are also arc-wise connected.

Let $\nu(t)$ be an arbitrary path in $L$, and $\nu(0)=b$. An arbitrary point $z \in p_{2}^{-1}(b)$ has a form $z=(a,\langle h\rangle, b), a \in L$. A homotopy $\kappa_{t}(s):=\left(\left.h \cdot \nu\right|_{[0, t]}\right)(s),(t, s) \in I \times I, I=[0,1]$, is situated in $L$. Hence $z(t):=\left(a,\left\langle\kappa_{t}\right\rangle, \nu(t)\right)$ is a path in the fibre $p_{1}^{-1}(a)$ such that $z=z(0)$ and $p_{2} \circ z(t)=\nu(t), t \in I$. Therefore the distribution $\mathcal{P}$ formed by tangent spaces to leaves of the submersion $\left.p_{1}\right|_{\mathbf{L}}: \mathbf{L} \rightarrow L$ is an integrable Ehresmann connection for the submersion $\left.p_{2}\right|_{\mathbf{L}}$.

Thus $\left(\mathbf{L},\left.p_{1}\right|_{\mathbf{L}},\left.p_{2}\right|_{\mathbf{L}}, L, L\right)$ is a simple transversal bifibration. The symmetry of the projections $p_{1}, p_{2}: * G_{\mathcal{Q}}(\mathcal{F}) \rightarrow M$ gives rise to the symmetry of the restrictions $\left.p_{1}\right|_{\mathbf{L}}$ and $p_{2} \mid \mathbf{L}$. 
According to Proposition 5 (ii) the map $p: \mathbf{L} \rightarrow L \times L:(x,\langle h\rangle, y) \mapsto(x, y)$ is a regular covering map. Using the definition of $\mathbf{L} \subset * G_{\mathcal{Q}}(\mathcal{F})$ it is not difficult to check that the group of the covering transformations $\Psi$ of $p$ is isomorphic to $* H_{\mathcal{Q}}(L)$.

\section{Generalized Ehresmann connection for foliation with singularities and its} holonomy. Let $(M, \mathcal{F})$ be a foliation with singularities and $M^{0}$ be the union of leaves of maximal dimension. Let $\mathcal{Q}$ be a generalized distribution on $M$ which is transversal to the foliation $\mathcal{F}$ (we use terminology introduced in Section 2). $\mathcal{Q}$ is called a generalized Ehresmann connection of $\mathcal{F}$ if for any admissible pair of paths $(\sigma, h)$, where $\sigma((0,1)) \subset$ $M^{0}$, there exists a v.h.h. with the base $(\sigma, h)$. Thus, we extend the notion of Ehresmann connection for foliations with singularities by considering only horizontal paths without singular points except for the endpoints.

Let $(M, \mathcal{F}, \mathcal{Q})$ be a singular foliation with a generalized Ehresmann connection. Then the distribution $\mathcal{Q}^{0}:=\left.\mathcal{Q}\right|_{M^{0}}$ is an Ehresmann connection for the regular foliation $\left(M^{0}\right.$, $\left.\mathcal{F}^{0}\right)$, where $\mathcal{F}^{0}:=\left.\mathcal{F}\right|_{M^{0}}$. Let $\Omega_{a}^{0}:=\left\{\sigma \in \Omega_{a} \mid \sigma((0,1]) \subset M^{0}\right\}$ and $\Omega_{a}^{*}:=\{\sigma \in$ $\left.\Omega_{a} \mid \sigma((0,1)) \subset M^{0}\right\}$ then $\Omega_{a}^{0} \subset \Omega_{a}^{*}$. If $\sigma \in \Omega_{a}^{0}$, each path $\sigma^{\prime}$ which is $\rho$-equivalent to $\sigma$ also lies in $\Omega_{a}^{0}$. Therefore $\Omega_{a}^{0} / \rho \subset \Omega_{a}^{*} / \rho$. For any admissible pair of paths $(\sigma, h)$, where $\sigma \in \Omega_{a}^{*}$, there exists a v.h.h. with the base $(\sigma, h)$. Let $\Phi_{a}^{*}\left([\sigma]_{\rho},[h]\right):=[\widetilde{\sigma}]_{\rho}$, where $[h] \in \pi_{1}(L, a), \sigma \stackrel{H}{\longrightarrow}>\widetilde{\sigma}$. Similar to the proof of Proposition 1 , one can check that $\Phi_{a}^{*}$ defines a right action of the fundamental group $\pi_{1}(L, a)$ on the quotient set $\Omega_{a}^{*} / \rho$. For a singular foliation with a generalized Ehresmann connection $(M, \mathcal{F}, \mathcal{Q})$, we call the quotient group $H_{\mathcal{Q}}(L, a):=\pi_{1}(L, a) / \operatorname{ker} \Phi_{a}^{*}$ the $\mathcal{Q}$-holonomy group of the leaf $L$ at $a \in L$.

In the case of a regular foliation $(M, \mathcal{F})$ with an Ehresmann connection $\mathcal{Q}$ we have $M=M^{0}$ and the group $H_{\mathcal{Q}}\left(L_{\alpha}, x\right)$ coincides with the $\mathcal{Q}$-holonomy group of $L_{\alpha}$ at $x \in L_{\alpha}$.

As $\operatorname{ker} \Phi_{a}^{*} \subset \operatorname{ker} \Phi_{a}$, we have the following assertion.

Proposition 7. For any leaf $L$ of a singular foliation $(M, \mathcal{F}, \mathcal{Q})$ with a generalized Ehresmann connection there exists a group epimorphism $\nu: * H_{\mathcal{Q}}(L, a) \rightarrow H_{\mathcal{Q}}(L, a)$ such that the diagram

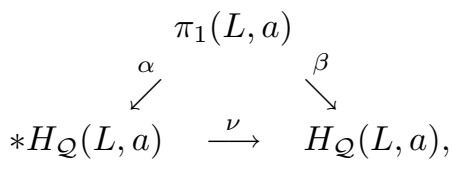

where $\alpha$ and $\beta$ are the quotient maps, is commutative.

Since $M^{0}$ is an open (possibly disconnected) submanifold in $M$, for any leaf $L \subset M^{0}$ we have the germ holonomy group $\Gamma(L, a), a \in L$, which is widely used in the foliation theory. Evidently, there exists a natural group epimorphism $\chi: H_{\mathcal{Q}}(L, a) \rightarrow \Gamma(L, a)$ such that $\chi \circ \beta=\gamma$, where $\gamma: \pi_{1}(L, a) \rightarrow \Gamma(L, a)$ is the projection, which maps $[h] \in \pi_{1}(L, a)$ to the germ of holonomy diffeomorphism corresponding to the path $h$. The following statement establishes a relation between different holonomy groups of a regular leaf $L$.

COROLlaRY 1. For any leaf $L \subset M^{0}$ of singular foliation with generalized Ehresmann connection the diagram 


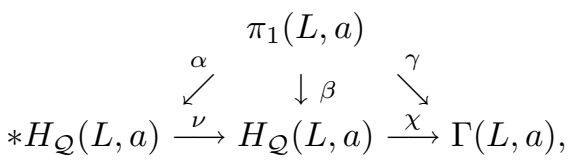

where $\alpha, \beta, \gamma$ are the quotient maps, and $\nu, \chi$ are the group epimorphisms, is commutative.

If for an admissible pair of paths $(\sigma, h)$ there exists a unique v.h.h. $H$ with the base $(\sigma, h), \sigma \stackrel{H}{\longrightarrow}>\widetilde{\sigma}, h \stackrel{H}{\longrightarrow}>\widetilde{h}$, we say that the curve $\widetilde{\sigma}$ is obtained by transfer of $\sigma$ along $h$, the path $\widetilde{h}$ is obtained by transfer of $h$ along $\sigma$, and denote it by $\sigma \stackrel{h}{\rightarrow}>\widetilde{\sigma}, h \stackrel{\sigma}{\longrightarrow}>\widetilde{h}$, as in the regular case.

\section{9. $\mathcal{Q}$-holonomy groupoid of foliations with generalized Ehresmann connec-} tions. Let $(M, \mathcal{F}, Q)$ be a foliation with generalized Ehresmann connection. If in the definition of $* G_{\mathcal{Q}}(\mathcal{F})$ we use the holonomy groups $H_{\mathcal{Q}}(L, x), L \in \mathcal{F}$, instead of the groups $* H_{\mathcal{Q}}(L, x)$ we shall get a groupoid $G_{\mathcal{Q}}(\mathcal{F})=\{(x,\{h\}, y)\}$, which will be called a $\mathcal{Q}$ holonomy groupoid. Topology in $G_{\mathcal{Q}}(\mathcal{F})$ is introduced similarly to the groupoid $* G_{\mathcal{Q}}(\mathcal{F})$.

Proposition 8. Let $(M, \mathcal{F}, Q)$ be a foliation with generalized Ehresmann connection. Then for any admissible pair $(\sigma, h)$, where $\sigma([0,1)) \subset M^{0}$ there exists a unique vertical horizontal homotopy $H$ with the base $(\sigma, h)$.

Proof. Given the horizontal curve $\sigma \in \Omega_{b}$ with $\sigma([0,1)) \subset M^{0}$, take a path $h$ in the leaf $L(b)$ such that $h(0)=b$. Since $\mathcal{F}$ is a foliation with a generalized Ehresmann connection $\mathcal{Q}$, there exists a v.h.h. $H$ with the base $(\sigma, h)$. Suppose that there exists another v.h.h. $K$ with the same base $(\sigma, h)$. For any $\tau \in(0,1)$, we have $\left.\sigma\right|_{[0, \tau]} \in \Omega_{b}^{0}$. In $M^{0}$ there exists the unique v.h.h. with the base $\left(\left.\sigma\right|_{[0, \tau]}, h\right)$, hence $\left.H\right|_{[0, \tau] \times I_{2}}=\left.K\right|_{[0, \tau] \times I_{2}}$, and then $\left.H\right|_{[0,1) \times I_{2}}=\left.K\right|_{[0,1) \times I_{2}}$. Since $H$ and $K$ are continuous, and $M$ is Hausdorff, we obtain that $H=K$.

The following lemma is analogous to Lemma 1.

LEMMA 2. If products of vertical paths $h \cdot k$ and $k^{\prime} \cdot g$ have common ends, then the following equalities are equivalent:

$$
\{h \cdot k\}=\left\{k^{\prime} \cdot g\right\} \text { and }\left\{k^{\prime-1} \cdot h\right\}=\left\{g \cdot k^{-1}\right\} .
$$

Let us consider the following conditions for a singular foliation $(M, \mathcal{F}, Q)$ with a generalized Ehresmann connection.

$\left(P_{1}\right)$ The union $M^{0}$ of leaves of maximal dimension is connected and dense in $M$.

$\left(P_{2}\right)$ For each point $c=(a,\{h\}, b)$ in $G_{\mathcal{Q}}(\mathcal{F})$ there is a neighbourhood $\mathcal{V}=\mathcal{V}\left(c, W_{1}, W_{2}\right)$ from the base $\Sigma$ of a topology in $G_{\mathcal{Q}}(\mathcal{F})$ defined above satisfying the following conditions:

(i) There is a dense saturated subset $M_{0}^{0}$ in $M^{0}$ such that a point $z=\left(x,\left\{t_{x} \cdot \tilde{h}\right.\right.$. $\left.\left.t_{y}^{-1}\right\}, y\right) \in \mathcal{V}$, where $x, y \in L_{\alpha} \subset M_{0}^{0}$ does not depend on the choice of paths $\sigma, t_{x}$, $t_{y}$ and v.h.h. with the base $\left(\sigma, h^{\prime}\right), h^{\prime} \in[h]$ used by us in the construction of $z$, i.e. $z$ is defined only by $x, y$ and $\{h\}$. 
(ii) There exists a fibred neighbourhood $\tilde{W}$ at point $b, W_{2} \subset \tilde{W}$ with the following property. Let $\left(\delta^{-1}, k\right)$ be an arbitrary admissible pair of paths in $W_{2}$, where $\delta \in \Omega_{b}^{0}$ and $k([0,1]) \subset M_{0}^{0}$. Let $\tilde{k}$ be the result of the unique transfer $k \stackrel{\delta^{-1}}{\longrightarrow}>\tilde{k}$ according to Proposition 8, with $\tilde{k}$ being a loop. Then the loop $\tilde{k}$ lies in $\tilde{W}$, i.e. $\tilde{k}(t) \in \tilde{W}$, $\forall t \in[0,1]$.

THEOREM 2. Let $(M, \mathcal{F}, Q)$ be a foliation with generalized Ehresmann connection satisfying $\left(P_{1}\right)$ and $\left(P_{2}\right)$. Then the following properties are fulfilled:

(i) The space of $\mathcal{Q}$-holonomy groupoid $G_{\mathcal{Q}}(\mathcal{F})$ is Hausdorff.

(ii) There exists a natural smooth structure on $\mathbf{L}:=p_{1}^{-1}(L), L \in \mathcal{F}$, and $\operatorname{dim} \mathbf{L}=$ $2 p, p=\operatorname{dim} L$, and the quintuple $\left(\mathbf{L},\left.p_{1}\right|_{\mathbf{L}},\left.p_{2}\right|_{\mathbf{L}}, L, L\right)$ is a symmetric simple transversal bifibration, and its structure group $\Psi$ is isomorphic to the holonomy group $H_{\mathcal{Q}}(L)$.

(iii) The map $f: * G_{\mathcal{Q}}(\mathcal{F}) \rightarrow G_{\mathcal{Q}}(\mathcal{F}):(x,\langle h\rangle, y) \mapsto(x,\{h\}, y)$ is a natural epimorphism of groupoids.

Proof. (i). Consider two different points $c_{1}=\left(a_{1},\left\{h_{1}\right\}, b_{1}\right)$ and $c_{2}=\left(a_{2},\left\{h_{2}\right\}, b_{2}\right)$ in $G_{\mathcal{Q}}(\mathcal{F})$. If $a_{1} \neq a_{2}$ (or $\left.b_{1} \neq b_{2}\right)$ there are fibred neighbourhoods $W_{1} \ni a_{1}, W_{1}^{\prime} \ni a_{2}$ such that $W_{1} \cap W_{1}^{\prime}=\emptyset$. Hence $\mathcal{V}\left(c_{1}, W_{1}, W_{2}\right) \cap \mathcal{V}\left(c_{2}, W_{1}^{\prime}, W_{2}^{\prime}\right)=\emptyset$.

Suppose that there are two different points $c_{1}=(a,\{h\}, b)$ and $c_{2}=(a,\{g\}, b)$ which are not separated. As by properties $\left(P_{1}\right)$ and (i) of $\left(P_{2}\right)$ the set $M_{0}^{0}$ is dense in $M$, there exists a sequence $\left\{z_{n}\right\} \subset p_{1}^{-1}\left(M_{0}^{0}\right)$ which converges both to $c_{1}$ and $c_{2}$. Hence for arbitrary fibred neighbourhoods $W_{1} \ni a$ and $W_{2} \ni b$ in $M$ there is a member of the sequence $z_{m} \in \mathcal{V}_{1} \cap \mathcal{V}_{2} \cap p_{1}^{-1}\left(M_{0}^{0}\right)$, where $\mathcal{V}_{1}:=\mathcal{V}\left(c_{1}, W_{1}, W_{2}\right)$ and $\mathcal{V}_{2}:=\mathcal{V}\left(c_{2}, W_{1}, W_{2}\right)$. Consider $\mathcal{V}_{1}$ and $\mathcal{V}_{2}$ satisfying the assumption $\left(P_{2}\right)$.

By the definition $\mathcal{V}_{1}$ point $z_{m} \in \mathcal{V}_{1} \cap p_{1}^{-1}\left(M_{0}^{0}\right)$ if and only if the following conditions are satisfied: 1) there is a horizontal curve $\sigma \in \Omega_{a}^{0}$ in $\left.W_{1} ; 2\right)$ there exists a vertical path $h^{\prime} \in[h]$ and a v.h.h. $H$ such that the result $\tilde{\sigma}$ of the transfer $\sigma \underset{h^{\prime}}{\stackrel{H}{\longrightarrow}}>\tilde{\sigma}, h^{\prime} \underset{\sigma}{\stackrel{H}{\rightarrow}}>\tilde{h}$; 3) $z_{m}=\left(x,\left\{t_{x} \cdot \tilde{h} \cdot t_{y}^{-1}\right\}, y\right)$. Similarly $z_{m}=\left(x,\left\{\tau_{x} \cdot \tilde{g} \cdot \tau_{y}^{-1}\right\}, y\right)$, where $\gamma \in \Omega_{a}^{0}$ in $W_{1}$ and there exist $g^{\prime} \in[g]$ and v.h.h. $H^{\prime}$ with the base $\left(\gamma, g^{\prime}\right)$ such that $\gamma \underset{g^{\prime}}{\stackrel{H^{\prime}}{\longrightarrow}}>\tilde{\gamma}, g^{\prime} \underset{\gamma}{\stackrel{H^{\prime}}{\longrightarrow}}>\tilde{g}$.

By the property (i) of $\left(P_{2}\right)$ without loss of generality we can assume that $\sigma=\gamma$ and $t_{x}=\tau_{x}$.

According to Lemma 2 the equality $\left\{t_{x} \cdot \tilde{h} \cdot t_{y}^{-1}\right\}=\left\{t_{x} \cdot \tilde{g} \cdot \tau_{y}^{-1}\right\}$ is equivalent to $\{\tilde{h}\}=\{\tilde{g} \cdot k\}$, where $k:=\tau_{y}^{-1} \cdot t_{y}$.

By Proposition 8 there exists a unique v.h.h. $H$ with the base $\left(\sigma^{-1}, \tilde{h}\right)$. As $\sigma \in \Omega_{a}^{0}$, so the equivalent class $\left[\tilde{\sigma}^{-1}\right]_{\rho}$ contains only one curve $\tilde{\sigma}^{-1}$. From this fact and the equality $\{\tilde{h}\}=\{\tilde{g} \cdot k\}$ it follows that the results of the transfers $\sigma^{-1}$ along $\tilde{h}$ and along $\tilde{g} \cdot k$ are the same and equal to $\tilde{\sigma}^{-1}$. Denote by $K$ the unique v.h.h. with the base $\left(\tilde{\gamma}^{-1}, k\right)$. Let $k \underset{\gamma^{-1}}{\stackrel{K}{\longrightarrow}}>\tilde{k}$, then $\tilde{k}$ is a loop in $b$ (see Fig. 5). According to the property (ii) of $\left(P_{2}\right)$ the path $\tilde{k}$ lies in fibred neighbourhood $\tilde{W}$, hence $\tilde{k}$ is a loop in $b$ belonging to the local leaf $l_{b}^{\tilde{W}}$. As $l_{b}^{\tilde{W}}$ is diffeomorphic to $\mathbf{R}^{q}$, the loop $\tilde{k}$ is homotopic to the constant path $e_{b}$ and $\left\{g^{\prime} \cdot \tilde{k}\right\}=\left\{g^{\prime}\right\}=\{g\}$. Therefore we have $\tilde{g} \cdot k \stackrel{\sigma^{-1}}{\longrightarrow}>g^{\prime} \cdot \tilde{k}$. Denote $\hat{g}:=g^{\prime} \cdot \tilde{k}$. Consider 


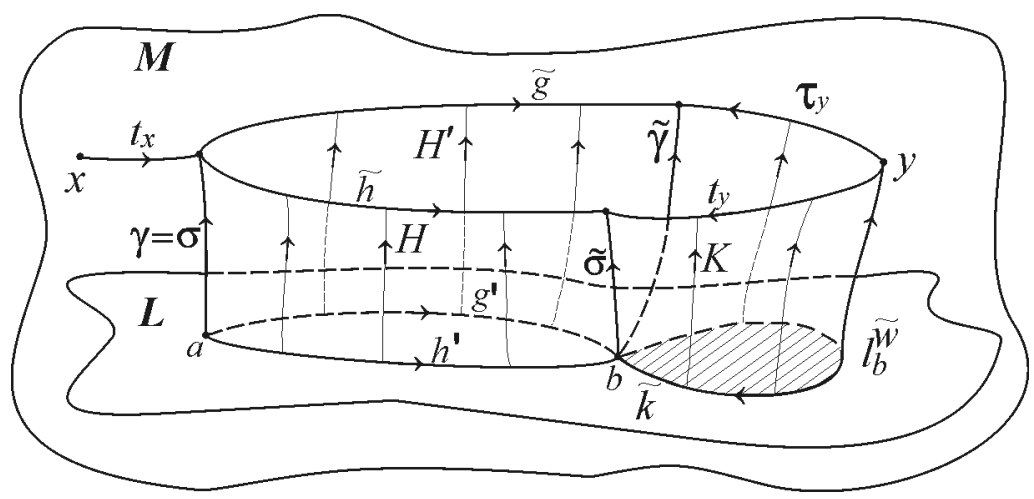

Fig. 5. $\{\tilde{k}\}=\left\{e_{b}\right\}$ and $\left\{g^{\prime} \cdot \tilde{k}\right\}=\left\{g^{\prime \prime}\right\}$

an arbitrary curve $\delta \in \Omega_{a}$. Let $\delta \underset{h^{\prime}}{\stackrel{\Psi}{\longrightarrow}}>\delta_{1}, \delta \underset{g^{\prime} \cdot \tilde{k}}{\stackrel{\Phi}{\longrightarrow}}>\delta_{2}$. V.h.hs $\Psi$ and $\Phi$ induce transfers of the product of path $\sigma^{-1} \cdot \delta$ along $\tilde{h}$ and along $\tilde{g} \cdot k$ with results $\tilde{\sigma}^{-1} \cdot \delta_{1}$ and $\tilde{\sigma}^{-1} \cdot \delta_{2}$, respectively. As $\{\tilde{h}\}=\{\tilde{g} \cdot k\}$ so $\left[\tilde{\sigma}^{-1} \cdot \delta_{1}\right]_{\rho}=\left[\tilde{\sigma}^{-1} \cdot \delta_{2}\right]_{\rho}$, hence $\left[\delta_{1}\right]_{\rho}=\left[\delta_{2}\right]_{\rho}$. Therefore $\{h\}=\left\{g^{\prime} \cdot \tilde{k}\right\}=\{g\}$, i.e. $c_{1}=c_{2}$ that is in contradiction with the assumption. Thus $G_{\mathcal{Q}}(\mathcal{F})$ is Hausdorff.

The proof of the statement (ii) is similar to the proof of Proposition 6 .

Applying Proposition 7 we get the assertion (iii).

Corollary 2. Let $(M, \mathcal{F}, Q)$ be a regular foliation with an Ehresmann connection. Then the manifold of the graph $G_{\mathcal{Q}}(\mathcal{F})$ is always Hausdorff.

Proof. From the proof of Proposition 4 it follows that the condition (i) of $\left(P_{2}\right)$ is fulfilled. In regular case in (ii) of $\left(P_{2}\right)$ as $\tilde{k}$ is a loop, $k$ is also a loop in $l_{y}^{W_{2}}$ and $\tilde{k}$ lies in $l_{b}^{W_{2}}$. Hence the condition (ii) of $\left(P_{2}\right)$ is true for $\tilde{W}=W_{2}$. Thus the conditions $\left(P_{1}\right)$ and $\left(P_{2}\right)$ are satisfied.

REMARK 3. The Hausdorff topological space $G_{\mathcal{Q}}(\mathcal{F})$ has a partition into manifolds $\left\{p_{1}^{-1}(L) \mid L \in \mathcal{F}\right\}$.

10. A global stability of compact leaves with finite $\mathcal{Q}$-holonomy. In this section we consider singular foliations with generalized Ehresmann connections $(M, \mathcal{F}, Q)$.

Consider the following condition:

$\left(P_{1}^{*}\right)$ The set $M^{0}$ of regular points is connected and for each singular leaf $L$ there is a horizontal curve $\sigma:[0,1] \rightarrow M$ such that $\sigma(0) \in L$ and $\sigma((0,1]) \subset M^{0}$.

Remark that the assumption $\left(P_{1}^{*}\right)$ implies $\left(P_{1}\right)$. The following theorem is proved by us in [34].

THEOREM 3. Let $(M, \mathcal{F}, Q)$ be a singular foliation with generalized Ehresmann connection, satisfying the condition $\left(P_{1}^{*}\right)$. If there exists a compact leaf $L_{0}$ in $M^{0}$ with a finite holonomy group $H_{\mathcal{Q}}\left(L_{0}\right)$ (or $\pi_{1}\left(L_{0}\right)$ ), then each leaf $L_{\alpha} \in \mathcal{F}$ is compact and has a finite holonomy group $H_{\mathcal{Q}}\left(L_{\alpha}\right)\left(\pi_{1}\left(L_{\alpha}\right)\right.$ respectively). 
This theorem can be considered as an analog of the famous Reeb theorem on global stability of a compact leaf $L_{0}$ with a finite group $\pi_{1}\left(L_{0}\right)$ of $C^{r}$-differentiable, $r \geq 2$, regular foliation of codimension one of a compact manifold.

11. Singular Riemannian foliations. Let $\mathcal{F}$ be a singular foliation on a Riemannian manifold $(M, g)$. The foliation $\mathcal{F}$ is called a Riemannian foliation if any geodesic of $g$ orthogonal to $\mathcal{F}$ at one point is orthogonal to $\mathcal{F}$ at any point of its domain. A foliated Riemannian manifold $(M, \mathcal{F}, g)$ is called transversally complete if the natural parameter on each horizontal geodesic changes over $(-\infty,+\infty)$.

The two following theorems belong to the author [29].

THEOREM 4. Let $(M, \mathcal{F}, g)$ be a transversally complete singular Riemannian foliation. Then:

1. The generalized orthogonal distribution $\mathcal{Q}=(T \mathcal{F})^{\perp}$ to $\mathcal{F}$ is an Ehresmann connection for $\mathcal{F}$.

2. The transfers with respect to $\mathcal{Q}$ keep the length of horizontal geodesics, i.e. if $\gamma \underset{h}{\longrightarrow}>$ $\tilde{\gamma}$ then $l(\tilde{\gamma})=l(\gamma)$.

A singular leaf $L$ is called tractable if $L$ has a saturated neighbourhood $W$ which satisfies the requirements:

1) $W$ is isomorphic to a fibre bundle over $L$ with the standard fibre $V$ (transversal to $\mathcal{F}$ ) endowed with the induced foliation $\Delta_{V}$;

2) the structure group of this fibre bundle is the group of automorphisms of the foliation $\Delta_{V}$, and the foliation on $W$, which is given locally as product of the leaf $L$ by $\Delta_{V}$, coincides with $\left.\mathcal{F}\right|_{W}$.

THEOREM 5. For a leaf $L$ of a transversally complete singular Riemannian foliation $\mathcal{F}$ the following conditions are equivalent:

1. $L$ is proper;

2. $L$ is closed as subset of $M$;

3. $L$ is tractable;

4. $L$ is locally stable.

COROLlaRY 3. Let $\mathcal{F}$ be as in Theorem 4 and $M$ be compact. Then each tractable leaf $L$ of $\mathcal{F}$ is also compact.

For regular Riemannian foliations Theorem 5 was proved by the author in [26]. Under an additional assumption about the existence of a complementary topological foliation $\mathcal{F}_{1}$, a local stability of a proper leaf had been proved by C. Ehresmann [6]. A parallel foliation on a Riemannian manifold admits the complementary orthogonal parallel foliation. Therefore such foliations are always regular and form a subclass of the class of previous foliations. For parallel foliations the equivalence of the conditions 1. - 3. was proved in [9], where the proof was considerably simpler, due to specificity of the case. 
12. The $\mathcal{Q}$-holonomy groupoid of a singular Riemannian foliation. In this section let $(M, \mathcal{F}, g)$ be a transversally complete singular Riemannian foliation. It has a natural Ehresmann connection $\mathcal{Q}=(T \mathcal{F})^{\perp}$. Let us consider $\mathcal{Q}$ as a generalized Ehresmann connection, where piece-wise horizontal geodesics play the role of horizontal curves.

Let $(M, \mathcal{F})$ be an orbit-like foliation in the sense of Molino relative to a smooth action of the compact group $O(m)$. It follows from the theory of compact transformation groups that the manifold $M^{0}$ of regular points is open connected and dense in $M$. Therefore for any transversally complete orbit-like singular Riemannian foliation $(M, \mathcal{F}, Q)$ conditions $\left(P_{1}\right)$ and $\left(P_{1}^{*}\right)$ are fulfilled and Theorem 3 is true.

THEOREM 6. Let $(M, \mathcal{F}, Q)$ be a singular transversally complete Riemannian foliation with a natural generalized Ehresmann connection $\mathcal{Q}=(T \mathcal{F})^{\perp}$, and suppose the condition $\left(P_{1}^{*}\right)$ is satisfied. Then the $\mathcal{Q}$-holonomy groupoid $G_{\mathcal{Q}}(\mathcal{F})$ is Hausdorff.

Proof. It is sufficient to check that property $\left(P_{2}\right)$ is satisfied and to apply Theorem 2 . Let us denote by $M_{0}^{0}$ the set of all leaves $L_{\alpha}$ of the regular foliation $\mathcal{F}^{0}:=\left.\mathcal{F}\right|_{M^{0}}$ with the trivial germ holonomy group $\Gamma\left(L_{\alpha}, x\right)$. According to the result of Epstein-Millett-Tischler [7] $M_{0}^{0}$ is a dense $G_{\delta}$-subset in $M^{0}$. As was shown by the author [27] there exists a canonical isomorphism of the groups $\Gamma\left(L_{\alpha}, x\right)$ and $H_{\mathcal{Q}^{0}}\left(L_{\alpha}, x\right)$ of the regular Riemannian foliation $\left(M^{0}, \mathcal{F}^{0}, \mathcal{Q}^{0}\right)$ with the Ehresmann connection $\mathcal{Q}^{0}:=\left.\mathcal{Q}\right|_{M^{0}}$. Therefore the $\mathcal{Q}^{0}$-holonomy group $H_{\mathcal{Q}^{0}}\left(L_{\alpha}, x\right)$ is also trivial.

Consider any point $c=(a,\{h\}, b) \in G_{\mathcal{Q}}(\mathcal{F})$ and its neighbourhood $\mathcal{V}=\mathcal{V}\left(c, W_{1}, W_{2}\right)$ from the base $\Sigma$ of the topology of $G_{\mathcal{Q}}(\mathcal{F})$. Let $z=\left(x,\left\{t_{x} \cdot \tilde{h} \cdot t_{y}^{-1}\right\}, y\right)$ be an arbitrary point in $\mathcal{V}$, and $x, y \in L_{\alpha} \subset M_{0}^{0}$. Here $\tilde{h}$ is the result of a transfer of $h^{\prime} \in$ $[h]$ along a horizontal curve $\sigma$ respective to v.h.h. $H$, i.e. $h^{\prime} \underset{\sigma}{\stackrel{H}{\rightarrow}}>\tilde{h}$, Suppose that $z=\left(x,\left\{\tau_{x} \cdot \hat{h} \cdot \tau_{y}^{-1}\right\}, y\right)$, where $h^{\prime \prime} \underset{\gamma}{\stackrel{K}{\longrightarrow}}>\hat{h}, h^{\prime \prime} \in[h], K$ is a v.h.h. with the base $\left(\gamma, h^{\prime \prime}\right)$. It is not difficult to show that a check of the condition (i) of $\left(P_{2}\right)$ is reduced to the case $h^{\prime \prime}=h^{\prime}$. As $H\left(I_{1} \times I_{2}\right) \cup K\left(I_{1} \times I_{2}\right)$ is a simply connected subset in $M$, so there are two paths $k_{1}$ from $\sigma(1)$ to $\gamma(1)$ in the local leaf $l_{x}^{W_{1}}$ and $k_{2}$ from $\tilde{\sigma}(1)$ to $\tilde{\gamma}(1)$ in $l_{y}^{W_{2}}$, where $\sigma \underset{h^{\prime}}{\stackrel{H}{\longrightarrow}}>\tilde{\sigma}, \gamma \underset{h^{\prime}}{\stackrel{K}{\longrightarrow}}>\tilde{\gamma}, h^{\prime} \underset{\gamma}{\stackrel{K}{\longrightarrow}}>\hat{h}$, such that $[\tilde{h}]=\left[k_{1}\right.$. $\left.\hat{h} \cdot k_{2}^{-1}\right]$. Remark that the paths $k_{1}$ and $t_{x}^{-1} \cdot \tau_{x}$ have common endpoints. By virtue of the equality $H_{\mathcal{Q}^{0}}\left(L_{\alpha}, x\right)=0$ and Proposition 8 we have $\left\{k_{1}\right\}=\left\{t_{x}^{-1} \cdot \tau_{x}\right\}$. Analogously, $\left\{k_{2}\right\}=\left\{t_{y}^{-1} \cdot \tau_{y}\right\}$. Therefore $\{\tilde{h}\}=\left\{t_{x}^{-1} \cdot \tau_{x} \cdot \hat{h} \cdot \tau_{y}^{-1} \cdot t_{y}\right\}$ and by Lemma 2 $\left\{t_{x} \cdot \tilde{h} \cdot t_{y}^{-1}\right\}=\left\{\tau_{x} \cdot \hat{h} \cdot \tau_{y}^{-1}\right\}$, i.e. $z$ depends only on $x, y$ and $\{h\}$. Hence the condition (i) of $\left(P_{2}\right)$ is true. Remark that the neighbourhoods $W_{1}$ and $W_{2}$ are as small as desired.

Let $(\tilde{W}, \tilde{\varphi})$ be a fibred chart at $b$ containing open $n$-dimensional ball $D_{2 \varepsilon}^{n}(b)$ with center $b$ and the radius $2 \varepsilon>0$ in Riemannian manifold $(M, g)$. Let $\left(W_{2}, \varphi\right)$ be a fibred chart at $b$, and $W_{2} \subset D_{\varepsilon}^{n}(b), \varphi=\left.\tilde{\varphi}\right|_{W_{2}}$. According to Theorem 4 transfers keep lengths of horizontal curves. Therefore the pair $W_{2} \subset \tilde{W}$ satisfies to the condition (ii) of $\left(P_{2}\right)$.

COROLlaRY 4. Let $(M, \mathcal{F}, \mathcal{Q})$ be a singular transversally complete Riemannian foliation with a natural generalized Ehresmann connection $\mathcal{Q}=(T \mathcal{F})^{\perp}$. If $(M, \mathcal{F})$ is an orbit-like 
foliation in the sense of Molino or if each singular leaf of $(M, \mathcal{F})$ is isolated in $M$, then the $\mathcal{Q}$-holonomy grupoid $G_{\mathcal{Q}}(\mathcal{F})$ is Hausdorff.

Corollary $5([23])$. The manifold of the graph of a regular Riemannian foliation $(M, \mathcal{F})$ on a complete Riemannian manifold $(M, g)$ with the bundle-like metric $g$ is Hausdorff.

\section{References}

[1] M. Bauer, Almost regular foliations, C. R. Acad. Sci. Paris 299 (1984), 387-390.

[2] B. Bigonnet and J. Pradines, Graphe d'un feuilletage singulier, C. R. Acad. Sci. Paris 300 (1985), 439-442.

[3] R. A. Blumenthal and J. J. Hebda, Ehresmann connections for foliations, Indiana Univ. Math. J. 33 (1984), 4, 597-611.

[4] P. Dazord, Holonomie des feuilletages singuliers, C. R. Acad. Sci. Paris 298 (1984), 27-30.

[5] C. Debord, Holonomy groupoids of singular foliations, J. Diff. Geom. 58 (2001), 467-500.

[6] C. Ehresmann, Structures feuilletées, in: Proc. Fifth Canad. Math. Cong., Montreal (1961), 109-172.

[7] D. B. A. Epstein, K. Millett and D. Tischler, Leaves without holonomy, J. London Math. Soc. 16 (1977), 548-552.

[8] R. Hermann, The differential geometry of foliations, Ann. of Math. 72 (1960), 445-457.

[9] V. Igosin and Ya. Shapiro, Stability of leaves of a foliation with a compatible Riemannian metric, Mat. Zametki 27 (1980), 767-778.

[10] S. Kashiwabara, The decomposition of differential manifolds and its applications, Tohoku Math. J. 11 (1959), 43-53.

[11] J. Kubarski, About Stefan's definition of a foliation with singularities: a reduction of the axioms, Bull. Soc. Math. France 118 (1990), 391-394.

[12] P. Molino, Riemannian Foliations, Progr. Math. 73, Birkhäuser, Basel, 1988.

[13] R. Palais, A global formulation of the Lie Theory of Transformation Groups, Mem. Amer. Math. Soc. 22 (1957).

[14] A. Piątkowski, A stability theorem for foliations with singularities, Dissertationes Math. (Rozprawy Mat.) 267 (1988).

[15] A. Piątkowski, The *-holonomy group of Stefan suspension of a diffeomorphism, Ann. Pol. Math. 58 (1993), 123-129.

[16] J. Pradines, Théorie de Lie pour les groupö̈des différentiables, C. R. Acad. Sci. Paris 263 (1966), 907-910.

[17] J. Pradines, Foliations: holonomy and local graphs, C. R. Acad. Sci. Paris 298 (1984), 297-300.

[18] Ya. L. Shapiro and N. I. Zhukova, Simple transveral bifibrations, Iz. VUZ. Mat. 1974, no. 4, 104-113.

[19] Ya. L. Shapiro and N. I. Zhukova, Global structure of reducible Riemannian manifolds, Iz. VUZ. Mat. 1980, no. 10, 60-62.

[20] P. Stefan, Accessible sets, orbits and foliations with singularities, Proc. London Math. Soc. 29 (1974), 699-713.

[21] H. J. Sussmann, Orbits of familities of vector fields and integrability of distributions, Trans. A.M.S. 180 (1973), 171-188.

[22] H. Suzuki, Holonomy groupoids of generalized foliations, I, Hokkaido Math. J. 19 (1990), $2,215-227$.

[23] H. Winkelnkemper, The graph of a foliation, Ann. Global Anal. Geom. 1 (1983), 51-75. 
[24] R. Wolak, Graphs, Ehresmann connections and vanishing cycles, in: Diff. Geom. and Appl. Proc. Conf., Brno, 1996, 345-352.

[25] R. Wolak, Connections for singular foliations on stratified manifolds, Publ. Math. Debrecen 63 (2003), 623-633.

[26] N. I. Zhukova, On the stability of leaves of Riemannian foliations, Ann. of Global Analysis and Geometry 5 (1987), 261-271.

[27] N. I. Zhukova, Foliations that are compatible with systems of paths, Iz. VUZ. Mat. 1989, no. $7,5-13$.

[28] N. I. Zhukova, Graph of a foliation with an Ehresmann connection and some its applicatons, VINITI, 1990, Dep. 1154-B90, 53 p.

[29] N. I. Zhukova, Stability criterion of leaves of Riemannian foliations with singularities, Iz. VUZ. Mat. 1992, no. 4, 88-91.

[30] N. I. Zhukova, The graph of a foliation with Ehresmann connection and stability of leaves, Iz. VUZ. Mat. 1994, no. 2, 78-81.

[31] N. I. Zhukova, Foliations with connections and Millett conjecture, Fundamental. problemy mat. i mekh., Matemat. Moscow State Univ. 1 (1994), 174-177.

[32] N. I. Zhukova and G. V. Chubarov, Aspects of the qualitative theory of suspended foliations, J. of Difference Equations and Applications 9 (2003), 393-405.

[33] N. I. Zhukova, Properties of graphs of Ehresmann connections, Vestnik Nezhegorodsk. Univ., Ser. Matem. 2004, no. 1, 73-87.

[34] N. I. Zhukova, Ehresmann connection of foliation with singularities and global stability of leaves, Iz. VUZ. Mat. 2004, no. 10, 45-56. 